\title{
Juliane Besters-Dilger
}

\section{Das Ende der Universität als Ort der Lehre?}

\begin{abstract}
Als Praktikerin möchte ich beginnen mit den Ergebnissen einer repräsentativen Umfrage unter Studierenden und Lehrenden der Universität Freiburg, die ich vor zwei Jahren zum Thema ,Digitale Lehre' durchgeführt habe: Studierende wünschen sich zu 80 Prozent mehr multimediale Lehrinhalte, unter anderem mehr Vorlesungsaufzeichnungen, mehr Videos, mehr Testmöglichkeiten zur Überprüfung des eigenen Lernfortschritts. Sie sprechen sich aber auch mit deutlicher Mehrheit gegen einen Ersatz der Präsenzlehre durch digitale Lehre aus; sie sehen Probleme bezüglich ausreichender Motivation, fehlender Lerndisziplin sowie die verstärkte Gefahr der Prokrastination (die sowieso eines der großen studentischen Probleme ist). Lehrende würden zu 80 Prozent gern mehr digitale Lehre anbieten - ein erstaunliches Ergebnis -, geben aber an, dies nur bei erhöhtem Ressourceneinsatz tun zu können: Sie brauchen vor allem Zeit zur Entwicklung digitaler Formate, bessere technische Ausstattung und Unterstützung, bessere Beratung. Auffallend ist die starke Differenzierung nach Fachkultur: die Technische Fakultät, die Fakultäten für Biologie und Medizin, die sowieso schon führend sind im Einsatz digitaler Lehre (einschließlich Virtual Reality), würden gerne mehr tun, andere Fakultäten zeigen kaum Bedarf. Wie lässt sich dieses Ergebnis in eine Strategie der Digitalisierung der Lehre integrieren?
\end{abstract}

Präsenzlehre bleibt ein fundamentales Bedürfnis der Studierenden, vor allem in den Anfangssemestern. In dieser Phase ist digitale Lehre daher Ergänzung, nicht Ersatz der Präsenzlehre. Bei doppeltem Angebot der Lehrveranstaltung (einmal Präsenzlehre, einmal z.B. Videoaufzeichnung) steht es den Studierenden frei, ob sie an beiden teilnehmen oder vielleicht nur an der digitalen Lehrveranstaltung; alle Untersuchungen (universitätsinterne Befragungen; Schulmeister \& Metzger 2018 usw.) zeigen aber, dass die Prüfungsergebnisse bei Nicht-Präsenz deutlich schlechter sind. Die besten Ergebnisse erzielen Studierende, die während der Vorlesung anwesend sind und zusätzlich die digitale Lehre zur Prüfungsvorbereitung nutzen.

2 Open Access. (C) 2020 Juliane Besters-Dilger, publiziert von De Gruyter. (c) BY-NC-ND Dieses Werk ist lizenziert unter der Creative Commons Attribution-NonCommercial-NoDerivatives 4.0 Lizenz. https://doi.org/10.1515/9783110673265-013 
Digitale Lehre dient der Flexibilisierung des Studiums, da Wissen zeit- und ortsungebunden erworben werden kann. Zum anderen ermöglicht sie, die Heterogenität der Studierenden zu bewältigen, die sich z.B. in nichtdeutscher Muttersprache, Berufstätigkeit neben dem Studium, nichttraditionellem Bildungsweg (Studium ohne Abitur), nicht-akademischem Elternhaus, Kinderbetreuungspflichten und Pflege von Angehörigen manifestiert. Die unbegrenzte Wiederholbarkeit ist für diese Studierenden ein großer Vorteil. Digitale Lehre muss, um erfolgreich zu sein, aber immer von entsprechenden Teletutoren zur Beantwortung von Fragen, Chats, Foren, Selbsttests, Lernmaterialien usw. begleitet sein. Sonst sind die Abbruchquoten zu hoch (vgl. die Erfahrung mit den Massive Open Online Courses der 2000er Jahre mit Absolventenquoten unter $10 \%)$.

Digitales Lernen setzt die Fähigkeit zu selbstreguliertem Lernen (Selbstlernkompetenz) voraus, die den Studierenden vermittelt werden muss, da sie sie von der Schule oft nicht mitbringen. Laut den Lehrenden der Universität Freiburg haben die Studienanfängerinnen und -anfänger große Probleme damit, Wissensgrundlagen zu erlernen; diese Probleme verstärken sich bei nur digital angebotener Lehre. Insbesondere die Entwicklung und das Aufrechterhalten von Lernmotivation, die Entwicklung einer Lernstrategie und die Vermeidung von Ablenkung gelten als schwierige Bausteine der Selbstlernkompetenz.

Die Hoffnung, durch digitale Lehre Personalkosten einzusparen, ist längst widerlegt. Im Gegenteil, gut gemachte digitale Lehre mit ansprechbaren Tutorinnen und Tutoren, Begleitmaterialien, Foren und Chats ist wesentlich teurer als Präsenzlehre. Für die Lehrenden erfordert sie drei Bausteine: Didaktik (die Didaktik digitaler Lehre ist anders als die der Präsenzlehre), technische Beratung/Unterstützung und technische Ausstattung. In der prekären finanziellen Lage der Hochschulen kann die Stärkung der digitalen Lehre nur über Projektmittel - mit dem bekannten 
Problem der fehlenden Nachhaltigkeit - finanziert werden oder über viele Jahre hinweg in kleinen Schritten aus dem Universitätshaushalt.

Angesichts der Halbwertzeit des Wissens besteht der Sinn des Studiums unter anderem darin, neben einem stets zu ergänzenden und weiterzuentwickelnden Wissen die Fähigkeit zu lebenslangem Lernen zu erwerben. Hinzu kommen fundamentale Kompetenzen wie Problemlösung, Informationsmanagement, Teamarbeit, Normen- und Werteerwerb usw. Einen besonderen Stellenwert haben kommunikative Kompetenzen wie Argumentieren, Standpunkte vertreten, Präsentieren, Überzeugen, Körpersprache beherrschen usw., die alle auf digitalem Wege schlechter zu erwerben sind als auf analogem. Absolventen- und Alumni-Befragungen zeigen zudem, dass die im Studium entstehenden persönlichen Netzwerke von erheblicher beruflicher Relevanz und intensiver als reine Netz-Bekanntschaften sind.

Im Hinblick auf die Nicht-Vorhersehbarkeit der Entwicklung des Arbeitsmarktes ist Data Literacy als eine grundlegende Kompetenz unverzichtbar und muss Bestandteil jedes Curriculums werden. Auch die Fähigkeit zu digitalem Lernen und zur digitalen Kooperation mit (auch internationalen) Partnern (vgl. Kirchherr 2019; Meyer-Guckel 2019) muss im Laufe des Studiums vermittelt werden. Oft wird vergessen, dass diese Kompetenzen erst einmal bei den Lehrenden in ausreichendem $\mathrm{Ma} ß$ entwickelt werden müssen: Viele Lehrende verfügen bisher nicht über Data Literacy.

Ganz besonders sind Data Literacy, Fähigkeit zu digitalem Lernen und zu digitaler Zusammenarbeit in der modular aufgebauten Weiterbildung gefragt, die schon lange als Pionier der digitalen Lehre gilt. In der Weiterbildung sehe ich auch die beste Möglichkeit, Menschen ohne formale Hochschulzulassung, aber mit praktischer Erfahrung einzubinden und so die Hochschulen zu öffnen (vgl. z.B. „Angewandte Ernährungswissenschaft - 
Gesundheit, Leistung, Sport“ an der Universität Freiburg; Voraussetzungen: Abgeschlossenes Hochschulstudium und mindestens ein Jahr Berufserfahrung oder eine abgeschlossene Berufsausbildung mit mindestens einem Jahr Berufserfahrung). Der Wissenschaftsrat stellt zur Weiterbildung fest: „Eine formale Durchlässigkeit zwischen beruflicher und akademischer Bildung wird inzwischen zwar von den meisten Hochschulen gewährleistet, oft beschränken sich die Aktivitäten der Hochschulen aber noch auf vereinzelte Angebote oder die Anerkennung von formal erworbenen Kompetenzen. Ein strategischer Ansatz zur Verbesserung der Durchlässigkeit, attraktive Angebotsstrukturen für Berufstätige und ein gut ausgebauter Weiterbildungsbereich sind allerdings noch selten“" (Wissenschaftsrat 2019, S. 36).

Die universitären Arbeitszeitmodelle sind zu überdenken. Viele Lehrende würden gern innovative digitale Lehrveranstaltungen entwickeln, haben aber ein zu hohes Lehrdeputat und zu viele administrative Aufgaben. Digitale Lehre zu konzipieren und umzusetzen ist wesentlich aufwändiger und kostet mehr Zeit als Präsenzlehre. Es darf nicht so sein, dass eine (partielle) Freistellung für die Entwicklung digitaler Lehrformate nur auf Kosten der Kolleginnen und Kollegen realisiert werden kann, d. h. zur Erhöhung von deren Lehrverpflichtung führt (so die Lehrverpflichtungsverordnung), vielmehr müssen Lehraufträge auf Kosten der Hochschule vergeben werden.

Der stark ausgeprägte Wunsch der Studierenden nach kollektivem Lernen kann nicht allein durch digitale Arbeitsgruppen erfüllt werden. Physische Nähe wird als elementares Bedürfnis empfunden. Die Raumkonzepte der Hochschulen sind entsprechend anzupassen (open spaces, makerspaces) und die betreffenden Vorgaben der deutschen Bundesländer zu überarbeiten. 
Digitale Lehre eignet sich hervorragend zum Wissensaustausch mit Partnerhochschulen in der ganzen Welt. Auf diese Weise kann die Hochschule ihr Lehrangebot erweitern, unter anderem in den so genannten Kleinen Fächern, die aufgrund geringer Personalressourcen oft kein attraktives, vielfältiges Lehrangebot anbieten können.

Digitale Lehre lässt sich nicht top down verordnen. Man kann aber Anreize schaffen und ein überfakultäres Netzwerk von engagierten und interessierten Lehrenden initiieren, die als Multiplikatorinnen und Multiplikatoren wirken. Ziel muss eine von allen Statusgruppen akzeptierte Digitalisierungsstrategie sein, die am besten in einem co-creation process erarbeitet wird.

Die Digitalisierung der Administration hinkt der Digitalisierung der Lehre hinterher. Wichtige Stichworte sind: Datenschutz, Datenarchivierung, eAkte, eRechnung, im Zusammenhang mit Lehre aber vor allem das Campus-Management (CMS) und das Studierendenmanagement (SMS), zu dem die digitale Prüfungsverwaltung gehört. Wenn Lehrende, die eine digitale Lehrveranstaltung durchgeführt haben, die Noten anschließend in Papierformulare eintragen müssen, damit diese von Mitarbeitenden des Prüfungsamts in digitale Formulare eingetippt werden, dann wird deutlich, dass die Digitalisierung der Hochschule noch in weiter Ferne liegt.

Wie sieht die Zukunft aus? Ich glaube nicht an das Ende der Universität als Institution der Lehre und den Übergang zu rein digitaler Lehre. Das Erlebnis Universität lässt sich nicht durch digitale Lehre ersetzen. Das Gespräch vor und nach der Lehrveranstaltung mit den Mitstudierenden, die Selbsteinschätzung im Vergleich zu anderen, der Aufbau sozialer Beziehungen, die Integration in das Netzwerk der Dozentin oder des Dozenten und vieles mehr gehören zu einer gelingenden Persönlichkeitsentwicklung. Teamorientierte aktivierende Lehre, die forschendes 
Lernen, Service learning, problemorientiertes Lernen usw. fördert, verschafft Erlebnisse, die digitales Lernen nicht ermöglicht. Die teilweise Digitalisierung der Lehre wird aber zu einer wachsenden Konkurrenz der Lehrenden untereinander führen, und diese kommt den Studierenden ohne Zweifel zugute.

\section{Referenzen}

Kirchherr, Julian et al.: Future Skills: Welche Kompetenzen in Deutschland fehlen. Stifterverband für die deutsche Wissenschaft. Essen 2019.

Meyer-Guckel, Volker et al.: Future Skills: Strategische Potenziale für Hochschulen. Stifterverband für die deutsche Wissenschaft. Essen 2019.

Schulmeister, Rolf, Metzger, Christiane: Das Studierverhalten im Bachelor. Zeitbudget-Analysen der Workload in 29 Bachelor-Stichproben. 2009-2018. Hamburg 2018.

Wissenschaftsrat: Empfehlungen zur hochschulischen Weiterbildung als Teil des lebenslangen Lernens. Vierter Teil der Empfehlungen zur Qualifizierung von Fachkräften vor dem Hintergrund des demografischen Wandels. Berlin 2019. 


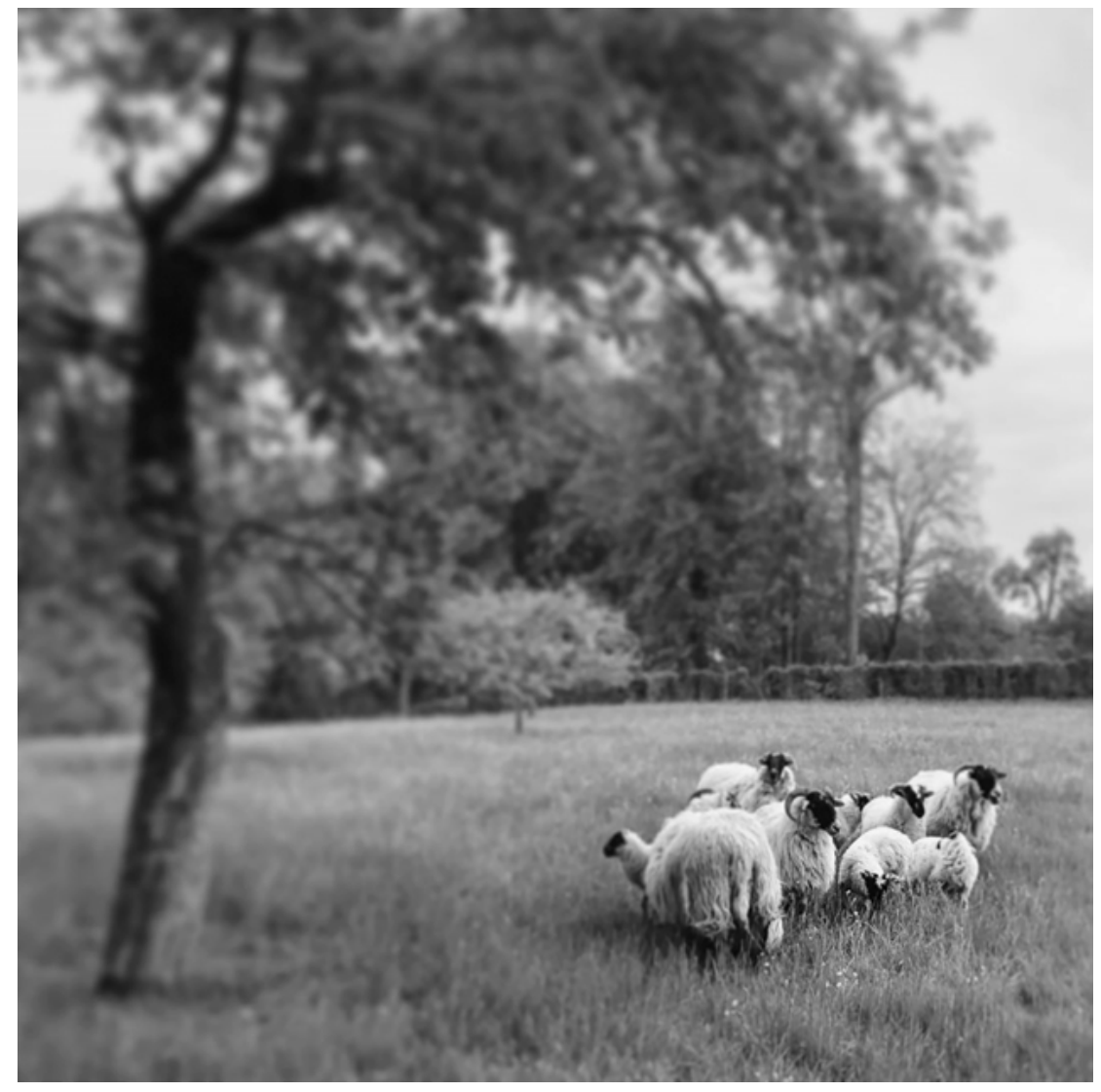


